\title{
1. Arbitrary-order Hilbert spectral analysis for time series possessing scaling statistics: a 2 comparison study with detrended fluctuation analysis and wavelet leaders
}

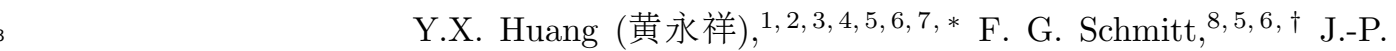 \\ 4 Hermand, ${ }^{7}$ Y. Gagne, ${ }^{9}$ Z.M. Lu (卢志明),${ }^{1,2,3}$ and Y.L. Liu (刘宇陆) ${ }^{1,2}$ \\ ${ }_{5}^{1}$ Shanghai Institute of Applied Mathematics and Mechanics, Shanghai University, Shanghai 200072, China \\ ${ }^{2}$ Shanghai Key Laboratory of Mechanics in Energy and Environment Engineering, Yanchang Road, Shanghai 200072, China \\ ${ }^{3}$ E-Institutes of Shanghai Universities, Shanghai University, Shanghai 200072, China \\ ${ }^{4}$ Université Lille Nord de France, F-59044 Lille, France \\ ${ }^{5}$ USTL, LOG, F-62930 Wimereux, France \\ ${ }^{6}$ CNRS, UMR 8187, F-62930 Wimereux, France \\ ${ }^{7}$ Environmental Hydroacoustics Laboratory, Université Libre de Bruxelles, \\ Avenue F-D. Roosevelt 50 - CP 194/5, B-1050 Brussels, Belgium \\ ${ }^{8}$ Univ Lille Nord de France \\ ${ }^{9}$ LEGI, CNRS/UJF/INPG, UMR 5519, F-38041 Grenoble, France
}

(Dated: October 17, 2018)

\begin{abstract}
In this paper we present an extended version of Hilbert-Huang transform, namely arbitrary-order Hilbert spectral analysis, to characterize the scale-invariant properties of a time series directly in an amplitude-frequency space. We first show numerically that due to a nonlinear distortion, traditional methods require high-order harmonic components to represent nonlinear processes, except for the Hilbert-based method. This will lead to an artificial energy flux from the low-frequency (large scale) to the high-frequency (small scale) part. Thus the power law, if it exists, is contaminated. We then compare the Hilbert method with structure functions (SF), detrended fluctuation analysis (DFA), and wavelet leader (WL) by analyzing fractional Brownian motion and synthesized multifractal time series. For the former simulation, we find that all methods provide comparable results. For the latter simulation, we perform simulations with an intermittent parameter $\mu=0.15$. We find that the SF underestimates scaling exponent when $q>3$. The Hilbert method provides a slight underestimation when $q>5$. However, both DFA and WL overestimate the scaling exponents when $q>5$. It seems that Hilbert and DFA methods provide better singularity spectra than SF and WL. We finally apply all methods to a passive scalar (temperature) data obtained from a jet experiment with a Taylor' s microscale Reynolds number $R e_{l} a m b d a \simeq 250$. Due to the presence of strong ramp-cliff structures, the SF fails to detect the power law behavior. For the traditional method, the ramp-cliff structure causes a serious artificial energy flux from the low-frequency (large scale) to the high-frequency (small scale) part. Thus DFA and WL underestimate the scaling exponents. However, the Hilbert method provides scaling exponents $\xi_{\theta}(q)$ quite close to the one for longitudinal velocity, indicating a less intermittent passive scalar field than what was believed before.
\end{abstract}

\section{INTRODUCTION}

18 Multifractal properties have been found in many fields, 19 such as turbulence [1 3, rainfall 4 4, financial time se20 ries [8], physiology [12, etc. Conventionally, multi${ }_{21}$ fractal properties of such time series are characterized 22 by the scaling exponents $\zeta(q)$, which are extracted by 23 structure function (SF) analysis: $\Delta V_{\ell}^{q} \sim \ell^{\zeta(q)}$, where ${ }_{24} \Delta V_{\ell}=|V(x+\ell)-V(x)|$ are the increment for scale sep25 aration $\ell$, and $\zeta(q)$ is a nonlinear function [1, 2, 13. The 26 function $\zeta(q)$ is linear for monoscaling processes and non27 linear for multifractal processes. We may also mention 28 the detrended fluctuation analysis (DFA) [14-16] or the 29 multifractal detrended fluctuation analysis [17], which 30 are sometimes also employed for scaling time series anal31 ysis. The DFA method is similar to SFs since it involves

\footnotetext{
* yongxianghuang@gmail.com

$\dagger$ francois.schmitt@univ-lille1.fr
}

32 increments and characterizes the scale invariance in the 33 physical domain.

Other widely used methods are wavelet-based meth35 ods, e.g. wavelet transform modulus maxima (WTMM), 36 wavelet leader (WL), or gradient modulus wavelet pro37 jection (GMWP), to extract the scaling exponents from 38 a scaling time series 18 30. However, as we will show in 39 this paper, the wavelets share the same drawback with ${ }_{40}$ Fourier transform, which requires high-order harmonic ${ }_{41}$ components to represent nonlinear processes.

42 Some of us have proposed recently a new methodology, 43 namely arbitrary-order Hilbert spectral analysis (HSA), 44 to characterize the scale invariant properties directly in 45 amplitude-frequency space $31-33$. It is an extended 46 version of the Hilbert-Huang transform (HHT), which 47 provides a joint probability density function (pdf) in an 48 amplitude-frequency space [31, 33. We have applied part 49 of this new methodology to several different time series 50 to show its efficiency and validity: turbulence experimen51 tal database 31, synthesized fractional Brownian mo- 
52 tion $(\mathrm{fBm})$ time series [32, surf zone marine turbulence 105 the local minima is zero [38, 39].

${ }_{53}$ data [34, and river flow discharge data [35. In this pa- 106 A subpart of the EMD algorithm, called "sifting pro${ }_{54}$ per we consider in length and precisely this new method ${ }_{107}$ cess," is then designed to decompose a given signal into ${ }_{55}$ and its validation and calibration. We first introduce 108 several IMF modes [38 40 . The first step of the sifting ${ }_{56}$ this new methodology in detail. We then validate and ${ }_{109}$ process is to identify all the local maxima (respectively, ${ }_{57}$ calibrate it by analyzing a synthesized multifractal time ${ }_{110}$ minima) points for a given time series $x(t)$. Once all the ${ }_{58}$ series. We finally consider a passive scalar (temperature) ${ }_{111}$ local extrema points are identified, the upper envelope ${ }_{59}$ data set with strong ramp-cliff structures. Due to the ${ }_{112} e_{\max }(t)$ and the lower envelope $e_{\min }(t)$ are constructed, 60 presence of ramp-cliff structures, the classical SF analy- ${ }_{113}$ respectively, for the local maxima and minima points by ${ }_{61}$ sis fails to detect the power law behavior [36. Addition- ${ }_{114}$ using a cubic spline algorithm. The mean between these 62 ally, for traditional methods, such as Fourier transform, 115 two envelopes is defined as

63 wavelet transform, high-order harmonics is required to

${ }_{64}$ represent these structures and leads to an artificial en${ }_{65}$ ergy flux from the large-scale part (low frequency) to the ${ }_{66}$ small-scale part (high frequency) 36 .

${ }_{67}$ This paper is organized as follows. We present the def${ }_{68}$ inition of the arbitrary-order Hilbert spectral analysis in ${ }_{69} \mathrm{Sec}$. II The classical structure function analysis, multi70 fractal detrended fluctuation analysis and wavelet leader 71 are also presented in this section. We then consider a 72 nonlinear effect by using the classical Duffing equation 73 to show the artificial high-order harmonic components ${ }_{74}$ required by the classical methods, for example, Fourier 75 transform, and wavelet transform, in Sec. III] In Sec. IV] 76 we perform a comparison study of the arbitrary-order 77 HSA with other methods by analyzing a fBm simula78 tion and a synthesized multifractal time series. We then 79 present in Sec. V Van analysis of real temperature data obso tained from a jet experiment. We finally draw the main 81 conclusions in Sec. VI

${ }_{85}$ The most innovative part of the Hilbert-Huang trans${ }_{86}$ form is the so-called empirical mode decomposition ${ }_{87}$ (EMD). In the real world most of the signals are multi88 components, which means that different scales can co89 exist simultaneously [37 39]. This may be considered 90 as fast oscillations superposed to slower ones at a local ${ }_{91}$ level [40, 41. Meanwhile, for decomposition methods, a ${ }_{92}$ characteristic scale (CS) is always defined implicitly or ${ }_{93}$ explicitly before the decomposition. For example, the ${ }_{94} \mathrm{CS}$ of the classical Fourier analysis is a period of sine ${ }_{95}$ wave. The CS of wavelet transform is the shape of the ${ }_{96}$ mother wavelet 33. In the present method, the CS is 13 97 defined as the distance between two successive maxima ${ }_{98}^{137}$ ation (SD), defined for two successive sifting processes
99 (respectively minima) points. Then the so-called intrin- ${ }_{138}$ as

99 sic mode functions (IMF) are proposed to represent each 100 mono-component signal. An IMF satisfies the following 101 two conditions: (i) the difference between the number of 102 local extrema and the number of zero-crossings must be 103 zero or one; (ii) the running mean value of the envelope 104 defined by the local maxima and the envelope defined by

$$
m_{1}(t)=\frac{e_{\max }(t)+e_{\min }(t)}{2}
$$

116 Thus the first component is estimated by

$$
h_{1}(t)=x(t)-m_{1}(t)
$$

${ }_{117}$ Ideally, $h_{1}(t)$ should be an IMF as expected. However, ${ }_{118} h_{1}(t)$ may not satisfy the above-mentioned conditions to ${ }_{119}$ be an IMF. The function $h_{1}(t)$ is then taken as a new 120 time series and this sifting process is repeated $j$ times, ${ }_{121}$ until $h_{1 j}(t)$ is an IMF

$$
h_{1 j}(t)=h_{1(j-1)}(t)-m_{1 j}(t)
$$

${ }_{122}$ The first IMF component $C_{1}(t)$ is then written as

$$
C_{1}(t)=h_{1 j}(t)
$$

${ }_{123}$ and the residual $r_{1}(t)$ as

$$
r_{1}(t)=x(t)-C_{1}(t)
$$

124 from the data $x(t)$. The sifting procedure is then re${ }_{125}$ peated on the residual until $r_{n}(t)$ becomes a monotonic 126 function or at most has one local extreme point, which ${ }_{127}$ means that no more IMF can be extracted from $r_{n}(t)$. ${ }_{128}$ There are finally $n-1$ IMF modes with one residual ${ }_{129} r_{n}(t)$. The original signal $x(t)$ is rewritten at the end of 130 the process as

$$
x(t)=\sum_{i=1}^{n-1} C_{i}(t)+r_{n}(t)
$$

131 To guarantee that the IMF modes retain enough physical ${ }_{132}$ sense, a certain stopping criterion has to be introduced to 133 stop the sifting process properly. Different types of stop${ }_{134}$ ping criteria have been introduced by several authors 38 $13540,42,43$. The first stopping criterion is a Cauchy-type 136 convergence criterion. We introduce the standard devi138 as

$$
\mathrm{SD}=\frac{\sum_{t=0}^{T}\left|h_{i(j-1)}(t)-h_{j}(t)\right|^{2}}{\sum_{t=0}^{T} h_{i(j-1)}^{2}(t)}
$$

${ }_{139}$ If a calculated $\mathrm{SD}$ is smaller than a given value, then 140 the sifting stops, and gives an IMF. A typical value is 
${ }_{141} 0.2 \sim 0.3$, proposed based on Huang et al.'s experiences 142 38, 39. Another widely used criterion is based on three ${ }_{143}$ thresholds $\alpha, \theta_{1}$, and $\theta_{2}$, which are designed to guarantee 144 globally small fluctuations meanwhile taking into account 145 locally large excursions [40]. The mode amplitude and ${ }_{146}$ evaluation function are given as

$$
a(t)=\frac{e_{\max }(t)-e_{\min }(t)}{2}
$$

147 and

$$
\sigma(t)=|m(t) / a(t)|
$$

${ }_{148}$ So that the sifting is iterated until $\sigma(t)<\theta_{1}$ for some 149 prescribed fraction $1-\alpha$ of the total duration, while ${ }_{150} \sigma(t)<\theta_{2}$ for the remaining fraction. The typical values 151 proposed by Rilling et al. 40 , are $\alpha \approx 0.05, \theta_{1} \approx 0.05$ 152 and $\theta_{2} \approx 10 \theta_{1}$, respectively based on their experience. In 153 our practice, if one of these criteria is satisfied, then the 154 sifting process will stop. We also set a maximal iteration 155 number (e.g., 300) to avoid over-decomposing the time 156 series.

${ }_{157}$ The above-described EMD algorithm performs the de158 composition on a very local level in the physical do159 main without a priori basis. This means that the 160 present decomposition is a posteriori: The basis is in161 duced by the data itself [38, 39, 41]. It is thus a 162 scale-based decomposition. Since its introduction, this 163 method has attracted large interests in various research ${ }_{164}$ fields: waves [34, 44, 45, biological applications 46165 48, financial studies 49, meteorology and climate stud166 ies [35, 50, 54, mechanical engineering [55, 56], acous${ }_{167}$ tics [57], aquatic environment [58, and turbulence [31, to 199 ${ }_{168}$ quote a few. More detail about the EMD algorithm can 169 be found in several methodological papers [38, 41, 43, 59].

171 After having extracted the IMF modes, one can ap172 ply the associated Hilbert spectral analysis to each com173 ponent $C_{i}$ in order to extract the energy time-frequency 174 information from the data $38,39,60$. The Hilbert trans175 form of a function $C(t)$ is written as

$$
\tilde{C}(t)=\frac{1}{\pi} P \int \frac{C\left(t^{\prime}\right)}{t-t^{\prime}} \mathrm{d} t^{\prime}
$$

176 where $P$ means the Cauchy principle value $37,38,60$, 17761 . For each mode function $C_{i}(t)$, one can then construct 178 the analytical signal 37,61 , $\mathbb{C}_{i}(t)$, as

$$
\mathbb{C}_{i}(t)=C_{i}(t)+j \tilde{C}_{i}(t)=\mathcal{A}_{i}(t) \mathrm{e}^{j \theta_{i}(t)}
$$

179 where

$$
\left\{\begin{array}{l}
\mathcal{A}_{i}(t)=\left[C_{i}(t)^{2}+\tilde{C}_{i}^{2}(t)\right]^{1 / 2} \\
\theta_{i}(t)=\arctan \left(\frac{\tilde{C}_{i}(t)}{C_{i}(t)}\right)
\end{array}\right.
$$

${ }_{180}$ Hence the instantaneous frequency is defined as

$$
\omega_{i}=\frac{1}{2 \pi} \frac{\mathrm{d} \theta_{i}(t)}{\mathrm{d} t}
$$

${ }_{181}$ The original signal is finally represented [excluding the 182 residual $\left.r_{n}(t)\right]$ as

$$
x(t)=\mathrm{R} \sum_{i=1}^{N} \mathcal{A}_{i}(t) e^{j \theta_{i}(t)}=\mathrm{R} \sum_{i=1}^{N} \mathcal{A}_{i}(t) e^{j \int \omega_{i}(t) d t}
$$

183 where " $\mathrm{R}$ " means real part. The above procedure is the ${ }_{184}$ classical Hilbert spectral analysis 37, 61. The combina185 tion of EMD and HSA is thus called Hilbert-Huang trans186 form by some authors [43. The Hilbert-Huang transform 187 can be taken as a generalization of the Fourier trans188 form, since it allows frequency modulation and ampli189 tude modulation simultaneously. The Hilbert spectrum, ${ }_{190} H(\omega, t)=\mathcal{A}^{2}(\omega, t)$, is designed to represent the energy 191 in a time-frequency representation 60. We further can 192 define the Hilbert marginal spectrum as

$$
h(\omega)=\int_{0}^{+\infty} H(\omega, t) \mathrm{d} t
$$

193 This is similar with the Fourier spectrum, and can be 194 interpreted as the energy associated with each frequency. 195 However, we underline the fact that the definition of fre196 quency here is different from the definition in the Fourier 197 frame. Thus the interpretation of the Hilbert marginal 198 spectrum should be given more caution [38, 39].

\section{9}

\section{Arbitrary-order Hilbert spectral analysis}

200 We can also define the joint pdf $p(\omega, \mathcal{A})$ of the instanta201 neous frequency $\omega$ and the amplitude $\mathcal{A}$ for each of these ${ }_{202}$ IMF modes 31, 33, 60. The Hilbert marginal spectrum 203 Eq. (14) is then rewritten as

$$
h(\omega)=\int_{0}^{+\infty} p(\omega, \mathcal{A}) \mathcal{A}^{2} \mathrm{~d} \mathcal{A}
$$

204 The above definition is no more than the second-order 205 statistical moment. This constatation has led some of 206 us to recently generalize this approach to arbitrary-order 207 moment $q \geq 0,3133$

$$
\mathcal{L}_{q}(\omega)=\int_{0}^{+\infty} p(\omega, \mathcal{A}) \mathcal{A}^{q} \mathrm{~d} \mathcal{A}
$$

208 In case of scale invariance, we have

$$
\mathcal{L}_{q}(\omega) \sim \omega^{-\xi(q)}
$$

209 in which $\xi(q)$ is the Hilbert-based scaling exponent func210 tion. Due to the integration operator, $\xi(q)-1$ can be ${ }_{211}$ associated with $\zeta(q)$ from SF analysis [31, 33.

A limitation of the Hilbert-based method we proposed ${ }_{213}$ here is that it lacks the ability to consider $q<0[62$. In 
214 other words, similarly with the SF analysis, it has no res- 260 where $\bar{x}$ is the mean value of $x$. We then divide it into ${ }_{215}$ olution on the right part of the singularity spectrum. The ${ }_{261} M_{n}$ segments of length $n(n<N)$ starting from both the 216 main drawback of the Hilbert-based method is its absence ${ }_{262}$ beginning and the end of the time series. Each segment 217 of solid theoretical ground, since the EMD part is almost ${ }_{263} v$ has its own local trend that can be approximated by ${ }_{218}$ empirical 43 . It has been found experimentally that ${ }_{264}$ fitting a $p$ th-order polynomial $P_{v}^{p}$ which is removed from 219 the method, especially for the HSA, is statistically stable 265 the data. The variances for all the segments $v$ and for all 220 with different stopping criteria [42]. Recently, Flandrin 266 segment lengths $n$ are then calculated by

221 et al. have obtained new theoretical results on the EMD 222 method [41, 59, 63 65. However, more theoretical work 223 is still needed to fully mathematically understand this 224 method.

\section{B. Structure function analysis}

226 The conventional way to extract scaling exponents is 227 the classical SF analysis, which has been proposed in the 228 field of turbulence and is now quite classical for intermit229 tency studies 13 . The $q$ th order SF is written as

$$
S_{q}(\ell)=\left\langle\left|\Delta x_{\ell}(t)\right|^{q}\right\rangle \sim \ell^{\zeta(q)}
$$

230 where $\Delta x_{\ell}(t)=x(t+\ell)-x(t)$ and $\ell$ is the time sepa231 ration. The scaling exponent $\zeta(q)$ characterizes the fluc232 tuation statistic at all scales; it is linear for monofractal 233 processes such as fractional Brownian motion, and non234 linear and concave (as a second Laplace characteristic 235 function) for multifractal processes 66. This approach 236 has been widely used in turbulent research [1, 2, 13] and ${ }_{237}$ also other research fields [9, 67, 68. However, the incre238 ment operation acts a filter and thus SF characterizes the 239 scale-invariant properties in an indirect way; see detailed 240 discussion in Refs. 33, 36.

${ }_{241}$ As we have shown elsewhere, the increment operation 242 in SF acts a filter and is a global operation. It thus 243 measures the scale invariant property in an indirect way. ${ }_{244}$ It is also found that it is strongly influenced by energetic ${ }_{245}$ large scale structures [33, 36. Therefore the SF analysis 246 is not suitable for those data which possess energetic large 247 scale structures. We will show an example of passive 248 scalar turbulence data with strong ramp-cliff structures 249 in Sec. V. More discussion can be found in Refs. [33, 36.

\section{Multifractal detrended fluctuation analysis}

251 DFA was first introduced by Peng et al. 14 to study 252 the scaling properties of DNA sequence, in which only 253 the second-order moment $q=2$ was considered. Later 254 this was generalized into a multifractal version by con255 sidering the arbitrary order $q$, namely multifractal de256 trended fluctuation analysis (MFDFA) [17, 69]. It then 257 became a more common technique for scaling data anal258 ysis [14 17, 69 75]. For a given discrete time series $x(i)$, ${ }_{259} i=1 \cdots N$, we first estimate its cumulative function

$$
Y(j)=\sum_{i=1}^{j}(x(i)-\bar{x}), \quad j=1, \cdots N
$$

$$
F^{2}(v, n)=\frac{1}{n} \sum_{j=1}^{n}\left\{Y[(v-1) n+j]-P_{v}^{p}(j)\right\}^{2}
$$

${ }_{267}$ The $q$ th-order fluctuation function is then defined as

$$
F^{q}(n)=\left(\frac{1}{2 M_{n}} \sum_{v=1}^{2 M_{n}}\left[F^{2}(v, n)\right]^{q / 2}\right)^{1 / q}
$$

${ }_{268}$ For discussion convenience, we redefine the $q$ th-order 269 fluctuation function as

$$
\mathcal{F}_{q}(n)=F^{q}(n)^{q}
$$

270 In case of scale invariance, we have power law scaling 271 within a significant range of $n$

$$
\mathcal{F}_{q}(n) \sim n^{h(q)}
$$

272 in which $h(q)$ is the corresponding scaling exponent func273 tion.

\section{D. Discrete wavelet transform and wavelet leaders}

Wavelets have been widely used in data analysis 276 and turbulence research [18, 23, 25, 27, 76, 78]. Several 277 wavelet-based methods have been proposed by several 278 researchers to extract the scaling exponents from a scal279 ing time series, for example, wavelet coefficients (WC), ${ }_{280}$ WTMM [18, 19, 76], WL [25, 26, 78, etc. We consider 281 here WC and WL.

${ }_{282}$ The discrete wavelet transform (DWT) is defined as

$$
\psi(k, j)=\int_{\mathbb{R}} x(t) \varphi\left(2^{-j} t-k\right) \mathrm{d} t
$$

283 where $\varphi$ is the chosen wavelet, $\psi(k, j)$ is the wavelet co284 efficient, $k$ is the position index, $j$ is the scale index, and ${ }_{285} \ell=2^{j}$ is the corresponding scale [77, 79]. The first way 286 to detect the scale-invariant properties is to consider the 287 wavelet coefficients

$$
Z_{q}(j)=\left\langle|\psi(k, j)|^{q}\right\rangle \sim 2^{j \tau(q)}
$$

288 where $\tau(q)$ are the corresponding scaling exponents.

${ }_{289}$ Every discrete wavelet coefficient $\psi(k, j)$ can be asso290 ciated with the dyadic interval $\varrho(k, j)$

$$
\varrho(k, j)=\left[2^{j} k, 2^{j}(k+1)\right)
$$


${ }_{291}$ Thus the wavelet coefficients can be represented as ${ }_{292} \psi(\varrho)=\psi(k, j)$. Wavelet leaders are defined as

$$
l(k, j)=\sup _{\varrho^{\prime} \subset 3 \varrho(k, j), j^{\prime} \leq j}\left|\psi\left(\varrho^{\prime}\right)\right|
$$

293 where $3 \varrho(k, j)=\varrho(k-1, j) \cup \varrho(k, j) \cup \varrho(k+1, j)$ [25, 28, 78, . 294 Thus power law behavior is expected

$$
\mathbb{Z}_{q}(j)=\left\langle l(k, j)^{q}\right\rangle \sim 2^{j \tau(q)}
$$

295 in which $\tau(q)$ is the corresponding scaling exponent. Its ${ }_{296}$ efficiency has been shown for various types of data set 297 25) 28, 78.

${ }_{298}$ Let us recall some previous comparison studies be299 tween WTMM, MFDFA and WL. Oświęcimka et al. 300 69. performed a comparison study between WTMM and 301 MFDFA by analyzing synthesized data. They stated that 302 the MFDFA provides a better estimation of singularity 303 spectrum than WTMM. Jaffard et al. 25] stated that WL ${ }_{304}$ provides a better singularity spectrum than WTMM. Ser${ }_{305}$ rano and Figliola 27. performed a comparison study be306 tween MFDFA and WL. They found that WL performs 307 better than MFDFA. However, for a short time series, 308 MFDFA is proposed to extract multifractal spectrum. A 309 detailed comparison can be found in Ref. 25], 69, 27, 310 respectively, for WTMM and WL, MFDFA and WTMM, 311 and WL and MFDFA.

312 However, we argue here that DWT violates two facts of 313 the time-frequency representation of a time series. First, 314 the scale of a time series from complex system, for exam315 ple, turbulent flows, is continuous in a statistical sense, 316 but not discrete on several scales [31, 33. The other one 317 is that for a certain scale, it may not exist all the time 318 33, 38, 61; see also the discussion in the next section. 319 Thus to represent a signal by using a DWT is not con320 sistent with the physical aspect.

\section{NONLINEAR EFFECTS}
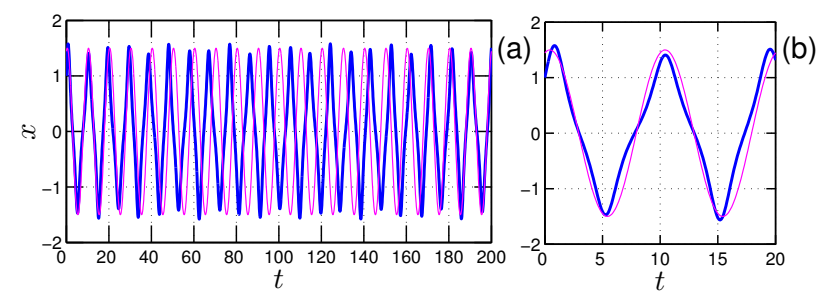

FIG. 1. (Color online) (a) a fifth order numerical solution (thick solid line) for Duffing equation, (b) An enlarged portion. For comparison, a sine wave with the same mean frequency is also shown as a thin solid line. The departure from a pure sine wave profile is the result of nonlinear interactions, which are nonlinear distortion.

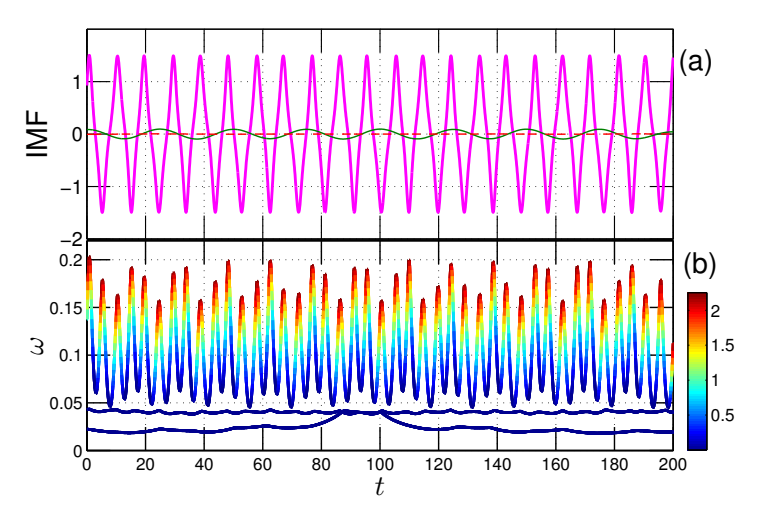

FIG. 2. (Color online) (a) The first three IMF modes from EMD; (b) the corresponding instantaneous frequency $\omega$ from Hilbert spectral analysis. Note the variation of the instantaneous frequency within one period. It is an intrawavefrequency-modulation, which corresponds to a nonlinear interaction. The instantaneous energy is encoded as a color.

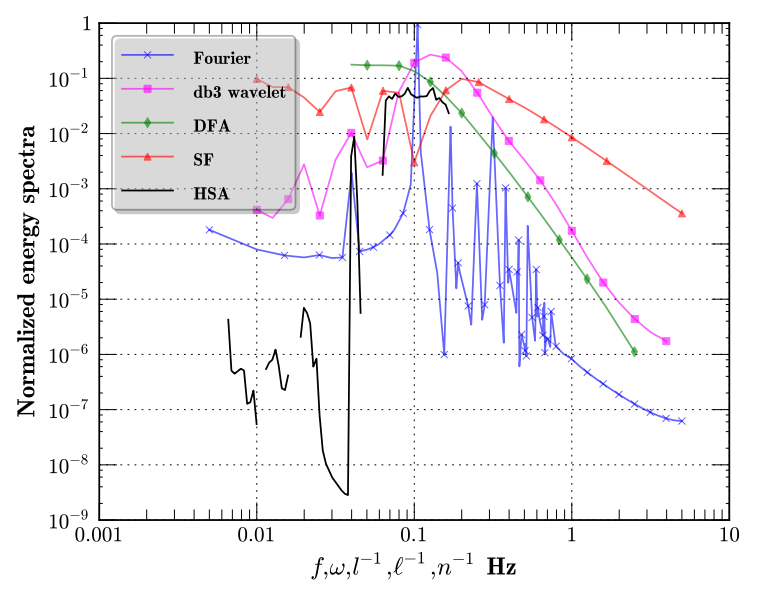

FIG. 3. (Color online) Energy spectra (the second-order statistical moments) provided by various methods: Fourier analysis $(\times)$, continuous wavelet transform with db3 wavelet $(\square)$, first-order DFA $(\diamond)$, SF $(\triangle)$ and Hilbert spectral analysis (solid line. The wavelet, SF and DFA spectra have been converted into spectral space by taking $f=1 / l, f=1 / \ell$ and $f=1 / n$, respectively. Except for the Hilbert-based method, all methods require high order harmonic components, which are not present in the original signal, see Figs. 11and 2 to represent this nonlinear process. The high-order harmonics may lead to an artificial energy flux from low frequencies (large scales) to high frequencies (small scales) in spectral space.

322 We first consider nonlinear effects by using the classical ${ }_{323}$ Duffing equation, which reads

$$
\frac{\mathrm{d}^{2} x}{\mathrm{~d} t^{2}}+x\left(1+\epsilon x^{2}\right)=b \cos (\Omega t)
$$

324 in which $\epsilon$ is a nonlinear parameter. It can be consid325 ered as a pendulum with forcing function $b \cos (\Omega t)$, in 326 which its pendulum length varies with the angle. Fig327 ure 1 shows a fifth-order Runge-Kutta numerical solu- 
328 tion (thick solid line) with $\epsilon=1, b=0.1, \Omega=2 \pi / 25,383 q$ th-order statistical moment) provided by SFs, MFDFA 329 and $\left[x(0), x^{\prime}(0)\right]=[1,1]$. The sampling frequency sets as 384 and wavelet are converted from the physical domain into ${ }_{330} 10 \mathrm{~Hz}$. For comparison, we also show a pure sine wave 385 the spectral domain by taking $f=1 / \ell, f=1 / n$, and 331 (thin solid line) with the same mean frequency. One can $386 f=1 / l$, respectively. The corresponding scaling expo332 see that the wave profile of the solution of the Duffing ${ }_{387}$ nents are estimated on the range $0.001<f<0.1$ (we set 333 equation is significantly deviating from a sine wave. This 388 here the sampling frequency as 1). Wavelet transform is 334 deviation is the result of nonlinear interaction, namely 389 performed by using the db3 wavelet. Due to the limita335 nonlinear distortion [38, 39. It is also clearly shown 390 tion of the SF analysis and the HSA, we only consider 336 that there are no high-order harmonic components in the 391 here the non-negative $q$ th-order moment, $q \geq 0$, the left 337 physical domain [33. Figure 2 shows the first three IMF 392 part of the singularity spectrum.

338 modes obtained from EMD decomposition and the cor-

339 responding instantaneous frequency $\omega$ from Hilbert spec-

340 tral analysis. The instantaneous energy is encoded as a 393

${ }_{341}$ color. The instantaneous frequency $\omega$ of the first IMF

342 mode is varying within one period. This corresponds to

${ }_{343}$ the so-called intrawave-frequency-modulation, which is

344 associated with the nonlinear interactions [33, 38, 39]. It

345 also clear shows that for a certain frequency, it may not 346 exist clearly all the time.

347 Figure 3 shows the normalized energy spectra (or the 348 second-order statistical moments) provided by various 349 methods: Fourier analysis $(\times)$, continuous wavelet trans350 form with db3 wavelet $(\square)$, the first-order DFA $(\diamond)$, ${ }_{351} \mathrm{SF}(\triangle)$ and HSA (solid line). For display convenience, 352 the wavelet, DFA and SF spectra have been converted 353 from physical domain into frequency domain by taking $354 f=1 / l, f=1 / n$ and $f=1 / \ell$, respectively. We empha355 size here that different wavelet families provide a simi356 lar spectral curve (not shown here). As pointed out by 357 Huang et al. 38 wavelet transform can be considered as 358 an adjustable window Fourier transform. Thus it inherits 359 the shortcomings of the Fourier transform. We observe 360 that except for the Hilbert spectral analysis, all methods 361 require high-order harmonic components to represent this 362 nonlinear process. High-order harmonic components are 363 not present in the time series (see Figs. 1 and 2 ). It is 364 thus a requirement of the method itself, not the physics 365 33, 38. This is the main drawback of traditional meth366 ods, in which the basis is given a priori. Therefore it 367 is inevitable that one requires high-order harmonic com${ }_{368}$ ponents to represent the difference between the analyzed 369 signal and the given basis. We argue here that high or370 der harmonic components may lead to an artificial energy 371 flux from low frequencies (large scales) to high frequen372 cies (small scales) in spectral space. Therefore, power 373 law behavior, if it exists, may be contaminated by this 374 artificial energy flux. We will show this point experimen375 tally by analyzing a temperature data set with strong 376 ramp-cliff structures in Sec. V

\section{IV. VALIDATION AND CALIBRATION}

378 In this section, we will validate the Hilbert-based 379 method by performing a comparison study of simulated $380 \mathrm{fBm}$ with Hurst number $H=1 / 3$ and synthesized multi381 fractal random walk with an intermittent parameter $\mu=$ 382 0.15. For comparison convenience, spectral curves (or the

\section{A. Fractional Brownian motion}

394 We have shown in previous works that the arbitrary395 order HSA can be applied to the fractional Brownian mo396 tion [31, 32]. Here we briefly recall these results. FBm 397 is a Gaussian self-similar process with a normal distribu398 tion increment, which is characterized by $H$, the Hurst 399 number $0<H<1$ [80 83]. Note that the singularity 400 spectra for the above mentioned methods are

$$
\alpha=\zeta^{\prime}(q), \quad f(\alpha)=\min _{q}\{\alpha q-\zeta(q)+1\}
$$

401 for SFs, and

$$
\alpha=\xi^{\prime}(q), \quad f(\alpha)=\min _{q}\{\alpha q-\xi(q)+2\}
$$

${ }_{402}$ for the Hilbert-based method, and

$$
\alpha=h^{\prime}(q)-1, \quad f(\alpha)=\min _{q}\{(\alpha+1) q-h(q)+1\}
$$

${ }_{403}$ for DFA, and

$$
\alpha=\tau^{\prime}(q)-1, \quad f(\alpha)=\min _{q}\{(\alpha+1) q-\tau(q)+1\}
$$

${ }_{404}$ for WC and WL, respectively. Ideally, we should have ${ }_{405} \alpha=H$ and $f(\alpha)=1$.

We performed 500 realizations each of length $2^{14}$ data ${ }_{407}$ points by applying a Fourier-based Wood-Chan algo408 rithm 84 with $H=1 / 3$, which corresponds to the Hurst 409 number of turbulent velocity. We apply the above men410 tioned methods to each realization of the data series. ${ }_{411}$ The final spectra and statistical errors are then estimated ${ }_{412}$ from these 500 realizations. Figure 4 show results for (a) ${ }_{413} \mathrm{SF}$ : (left) $S_{q}(\ell)$ with $q=0(\bigcirc), q=2(\square), q=4(\triangle)$ and ${ }_{414} q=6(\nabla)$, (middle) the corresponding scaling exponents ${ }_{415} \zeta(q)$ on the range $0 \leq q \leq 8$, (right) the correspond416 ing singularity spectrum $f(\alpha)$, (b) HSA, (c) DFA, and ${ }_{417}$ (d) wavelet, respectively. The symbols are the same as ${ }_{418}$ the SF symbols. Graphically, all methods provide com419 parable estimation of $f(\alpha)$. However, we note that the ${ }_{420}$ Hilbert-based method slightly overestimates $\xi(q)$ when ${ }_{421} q>6$. Additionally both the first- and second-order DFA ${ }_{422}$ provide slight underestimation of $h(q)$ and seem to pre${ }_{423}$ dict a systematic underestimation of the Hurst number ${ }_{424} H$. The WC and WL provide almost the same estimation 

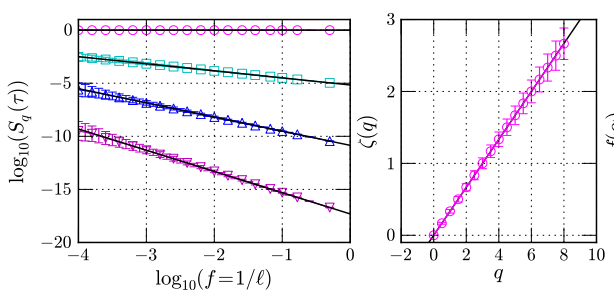

(a)
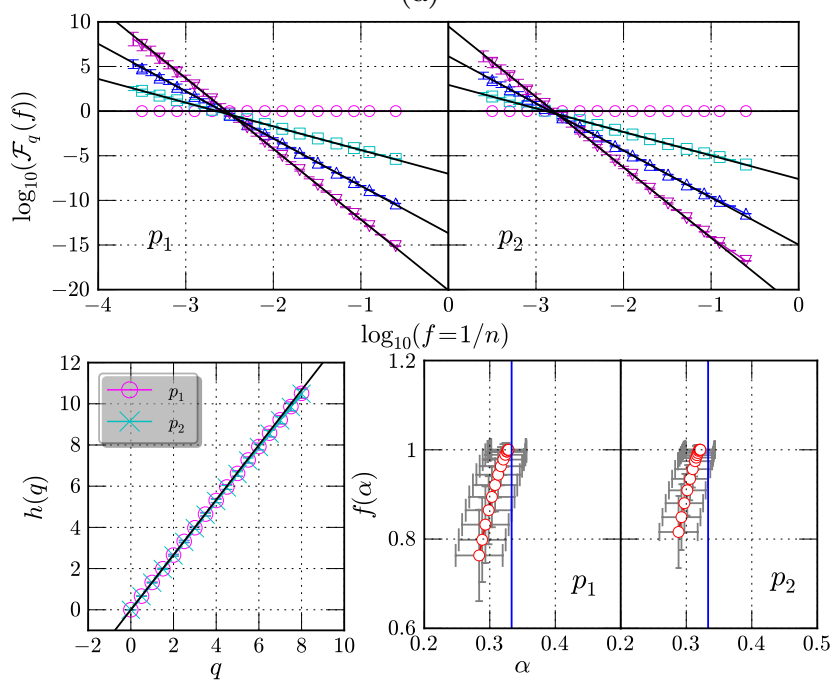

(c)
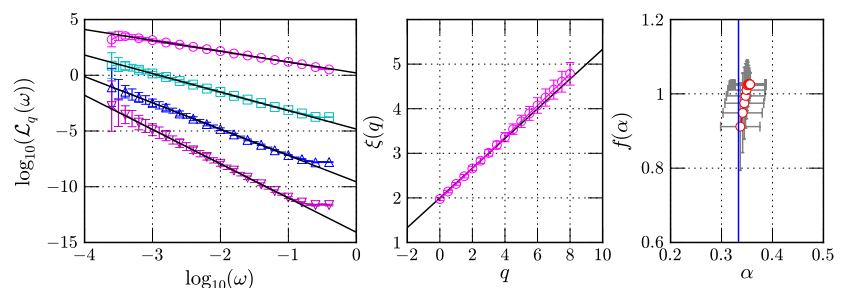

(b)
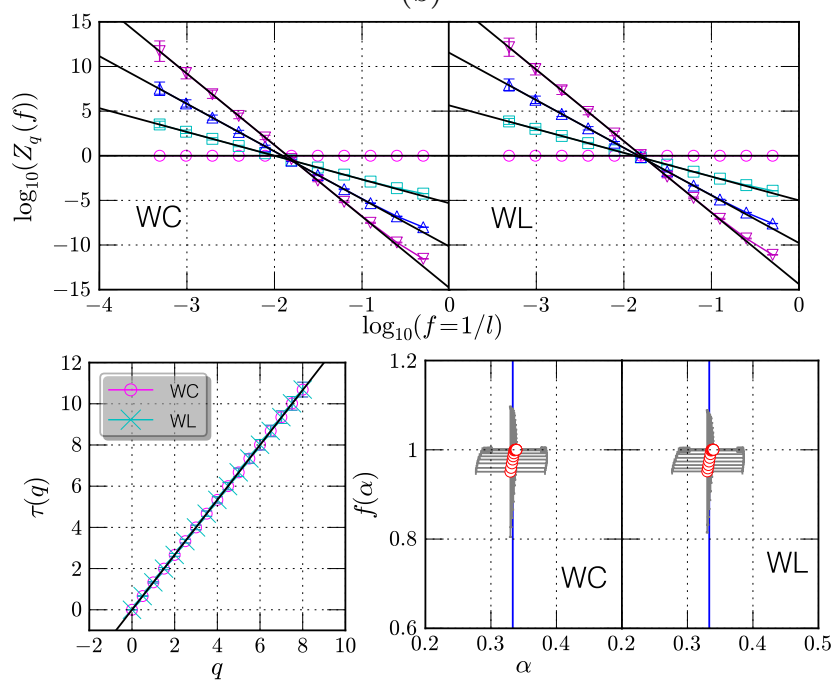

(d)

FIG. 4. (Color online) Analysis results of fBm with Hurst number $H=1 / 3$. (a) structure functions. left: $S_{q}(\ell)$ with $q=0$ $(\bigcirc), q=2(\square), q=4(\triangle)$ and $q=6(\nabla)$; middle: the corresponding scaling exponents $\zeta(q)$ on the range $0 \leq q \leq 8$; right: the corresponding singularity spectrum $h(\alpha)$. (b) results of Hilbert spectral analysis. (c) multifractal detrended fluctuation analysis (d) wavelet coefficients and wavelet leaders. The symbols are the same as for structure functions. Scaling exponents are estimated in the range $-3<\log _{10}(f)<-1$. The statistical errors are estimated from a total of 500 realizations.

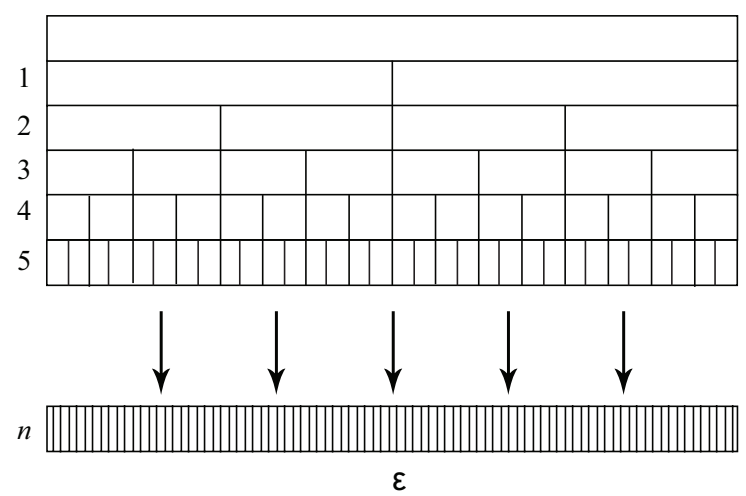

FIG. 5. Illustration of the discrete cascade process. Each step is associated with a scale ratio of 2 . After $n$ steps, the total scale ratio is $2^{n}$.

425 for this simple monofractal process. It seems that they ${ }_{426}$ provide a better estimation than Hilbert and DFA meth427 ods. This result is not in full agreement with Oświęcimka ${ }_{428}$ et al. 69], who stated that the MFDFA provides a better 429 estimation of $H$ than WTMM.

${ }_{430}$ The above results show that all methods provide com- ${ }_{432} H=1 / 3$. However, it seems that $\mathrm{SF}$ and wavelet based ${ }_{431}$ parable prediction of singularity spectra for $\mathrm{fBm}$ with ${ }_{433}$ methods provide a better estimation. 

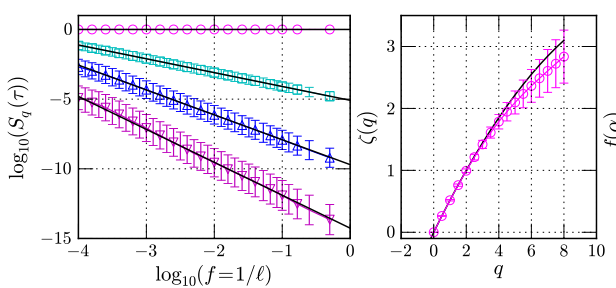

(a)
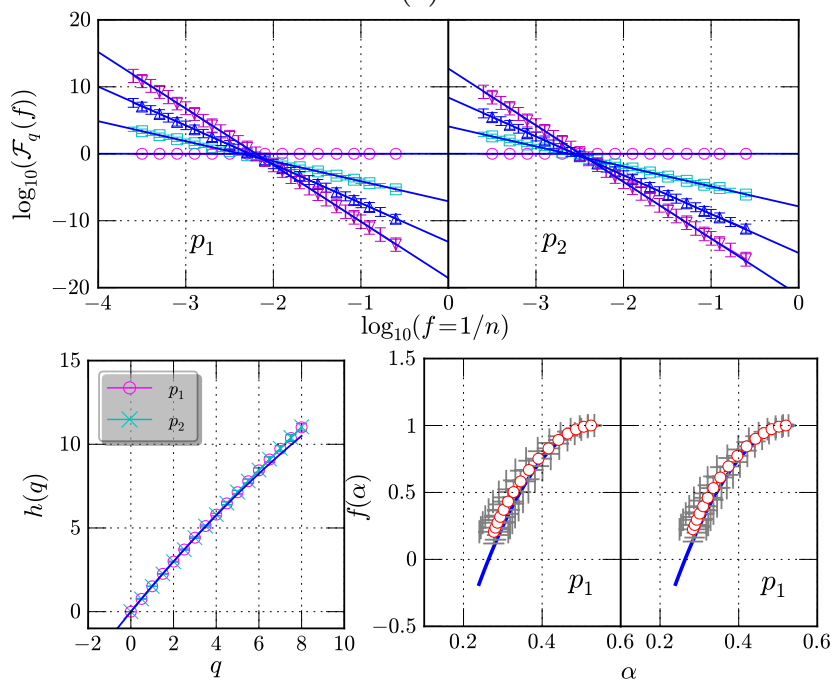

(c)
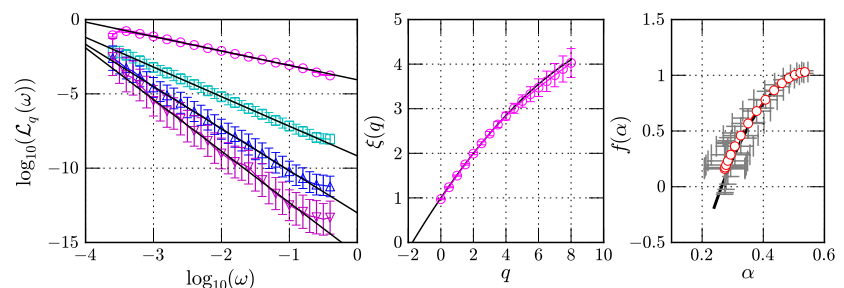

(b)
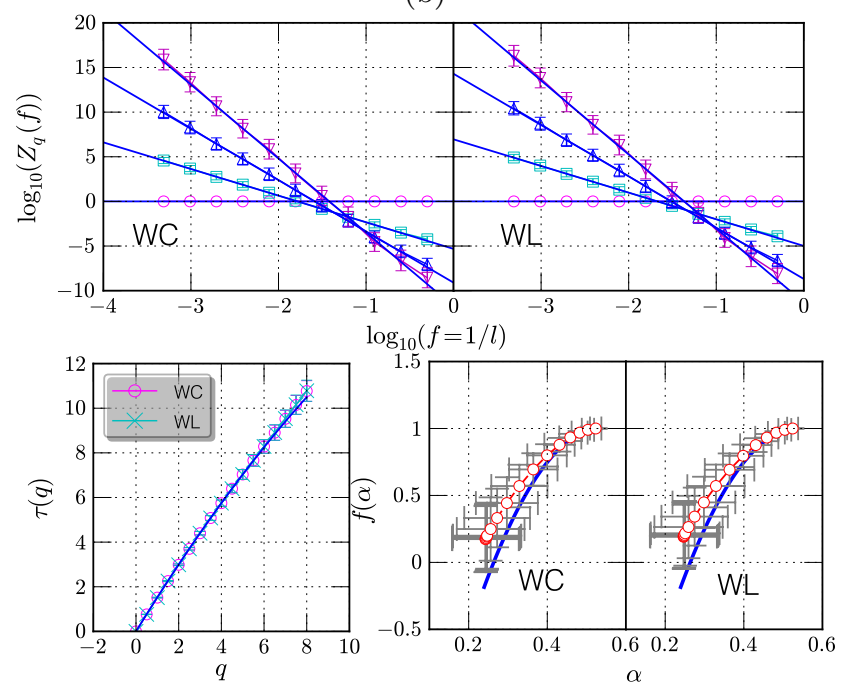

(d)

FIG. 7. (Color online) Multifractal random walk with $\mu=0.15$. (a) Structure function , (b) Hilbert spectral analysis, (c) multifractal detrended fluctuation analysis and $(\mathrm{d})$ wavelet coefficients and wavelet leaders. The symbols are the same as in Fig. 4 The statistical error bars are estimated from the total 100 realizations.

434

435

We show now that the new method applies to multi436 fractal time series. First, let us consider a multiplica${ }_{437}$ tive discrete cascade process to simulate a multifractal 438 measure $\epsilon(x)$. Figure 5 illustrates the cascade process 439 algorithm. The larger scale corresponds to a unique cell ${ }_{440}$ of size $L=\ell_{0} \lambda_{1}^{n}$, where $\ell_{0}$ is a fixed scale and $\lambda_{1}>1$ ${ }_{441}$ is a dimensional scale ratio. For discrete models, this ${ }_{442}$ ratio is often taken as $\lambda_{1}=2$. The models being dis${ }_{443}$ crete, the next scale involved corresponds to $\lambda_{1}$ cells, ${ }_{444}$ each of size $L / \lambda_{1}=\ell_{0} \lambda_{1}^{n-1}$. This is iterated and at ${ }_{445}$ step $p(1 \leq p \leq n)$ there are $\lambda_{1}^{p}$ cells, each of size ${ }_{446} L / \lambda_{1}^{p}=\ell_{0} \lambda_{1}^{n-p}$. There are $n$ cascade steps, and at step ${ }_{447} n$ there are $\lambda_{1}^{n}$ cells, each of size $\ell_{0}$, which is the smallest ${ }_{448}$ scale of the cascade. To reach this scale, all intermedi449 ate scales have been involved. Finally, at each point the ${ }_{450}$ multifractal measure writes as the product of $n$ cascade ${ }_{451}$ random variables

$$
\epsilon(x)=\prod_{p=1}^{n} W_{p, x}
$$

${ }_{452}$ where $W_{p, x}$ is the random variable corresponding to po${ }_{453}$ sition $x$ and level $p$ in the cascade 85. Following mul454 tifractal random walk ideas [86, 87, we generate a non-
455 stationary multifractal time series as

$$
u(x)=\int_{0}^{x} \epsilon\left(x^{\prime}\right)^{1 / 2} \mathrm{~d} B\left(x^{\prime}\right)
$$

456 where $B(x)$ is Brownian motion. Taking lognor${ }_{457}$ mal statistic for $\epsilon$, the scaling exponent $\zeta(q)$ such as ${ }_{458}\left\langle\left|\Delta u_{\tau}(t)\right|^{q}\right\rangle \sim \tau^{\zeta(q)}$ can be shown to be written as

$$
\zeta(q)=\frac{q}{2}-\frac{\mu}{2}\left(\frac{q^{2}}{4}-\frac{q}{2}\right)
$$

459 where $\mu$ is the intermittency parameter $(0 \leq \mu \leq 1)$ 460 characterizing the lognormal multifractal cascade.

Synthetic multifractal time series are generated follow462 ing Eq. 32 . For each realization, we choose $n=17$ lev${ }_{463}$ els, corresponding to data sets with data length 131, 072 ${ }_{464}$ points each. A sample for one realization is shown in ${ }_{465}$ Fig. 6 (a) for the multifractal measure and (b) for the 466 nonstationary multifractal time series with $\mu=0.15$. ${ }_{467}$ We perform 100 realizations with intermittent parameter ${ }_{468} \mu=0.15$. Except for the structure functions, we apply (31) 469 all methods to each realization by dividing one realiza470 tion into eight subsets with $2^{14}$ data points each. The 471 spectra for each realization are averaged over these eight 472 subsets. The final spectra and error bars are respectively 473 ensemble average and standard deviation estimated from 474 these 100 realizations. 
${ }_{475}$ Figure 7 shows the results of (a) SF, (b) HSA, (c) ${ }_{476} \mathrm{MFDFA}$ and (d) WC and WL, respectively. The sym477 bols are the same as in Fig. 4. The theoretical scaling 478 exponents and the corresponding singularity spectrum 479 $f(\alpha)$ on the range $0<q<8$ are shown as a solid line in 480 the corresponding sub figures. We see that SFs under${ }_{481}$ estimate $\zeta(q)$ when $q>4$. The corresponding estimated 482 singularity spectrum $f(\alpha)$ deviates from the theoretical ${ }_{483}$ line when $\alpha<0.4$, corresponding to $q>2.5$. It also has 484 the largest statistical error. Hilbert methodology slightly 485 underestimates $\xi(q)$ when $q>5$. It provides a better esti486 mation of scaling exponents and $f(\alpha)$ than SFs. MFDFA ${ }_{487}$ provides the smallest statistical errors for spectral curves ${ }_{488} \mathcal{F}_{q}(n)$, scaling exponents $h(q)$ and singularity spectrum ${ }_{489} f(\alpha)$. However, it still slightly overestimates $h(q)$ when ${ }_{490} q>6$. We note that the first- and second-order DFA 491 provide an equivalent result. WC and WL predict al${ }_{492}$ most the same spectral curves, scaling exponents $\tau(q)$ 493 and singularity spectrum $f(\alpha)$. The corresponding sin494 gularity spectrum significantly deviates from the theo495 retical curve. We also note that none of these methods 496 recover the whole theoretical line on the range $0<q<8$.

\section{V. PASSIVE SCALAR TURBULENCE WITH ${ }_{498}$ RAMP-CLIFF STRUCTURES}

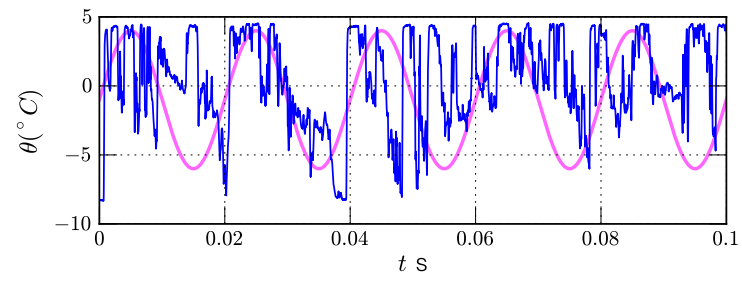

FIG. 8. (Color online) A $0.1 \mathrm{~s}$ portion of temperature data showing strong ramp-cliff structures. For comparison, a pure sine wave is also shown as a thick solid line. Note that the ramp-cliff structure is significantly different from a sine wave, which may cause serious artificial energy flux in Fourier spectral space.

499 We now apply the above-mentioned methods to a real 500 time data set, a temperature time series as a turbulent 501 passive scalar. The data are obtained from a jet experi502 ment performed at Joseph Fourier University Grenoble, ${ }_{503}$ France. The bulk Reynolds number is about $R e \simeq 60000$. ${ }_{504}$ The corresponding Taylor's microscale Reynolds number 505 is about $R e_{\lambda} \simeq 250$. The initial temperature of the two 506 flows are $T_{J}=27.8^{\circ} \mathrm{C}$ and $T=14.8^{\circ} \mathrm{C}$. The measure- 535 ${ }_{507}$ ment location is in the mixing layer and close to the noz- 5 508 zle of the jet. The sampling frequency is $50 \mathrm{kHz}$. The 537 a Kolmogorov- Obukhov-Corrsin 93 95] nonintermittent 509 total data length is $10 \mathrm{~s}$, corresponding to 500,000 data 538 scaling exponent $5 / 3$ for Hilbert spectrum, Fourier power 510 points. Figure 8 shows a 0.1 s portion temperature data, 539 spectrum, 8/3 for WL and DFA, and $2 / 3$ for SF, respec511 illustrating strong ramp-cliff structures. For comparison, 540 tively. Except for the SF, all methods display a clear 512 a pure sine wave is also shown. Obviously, the so-called ${ }_{541}$ power law on the range $80<f<2000 \mathrm{~Hz}$ or $100<$ 513 ramp-cliff structure is a large-scale structure with a very ${ }_{542} f<1000 \mathrm{~Hz}$, a more than one decade inertial range. ${ }_{514}$ sharp interface 88 91. We note that the profile of ramp- ${ }_{543}$ The corresponding scaling exponents are $\xi_{\theta}(2) \simeq 1.70$
FIG. 9. (Color online) Energy spectra (or the second-order statistical moment) provided by several methods. The inset shows the compensated spectra by multiplying the result by $f^{5 / 3}$ for Hilbert and Fourier, $f^{8 / 3}$ for DFA and wavelet, and $f^{2 / 3}$ for SFs, respectively. For clarity, the curves have been vertically shifted. Both Fourier and Hilbert methods predict a clear power law on the range $80<\omega<2000 \mathrm{~Hz}$. Due to the presence of ramp-cliff structures, the SF analysis fails to capture the power law behavior and DFA and wavelet predict a short inertial range on the range $100<f<1000 \mathrm{~Hz}$. The corresponding scaling exponents are $\beta_{\theta}=1.56$ for Fourier, $\xi_{\theta}(2)=1.70$ for Hilbert, $\tau_{\theta}(2)=2.46$ for WL with db3 wavelet, and $h_{\theta}(2)=2.47$ for the first-order DFA, respectively.

515 cliff structures is significantly deviating from a sine wave. 516 Thus for the Fourier-based methodologies, it is inevitable 517 that one requires high-order harmonic components to 518 represent their difference, in which the underlying idea 519 is a linear asymptotic approximation [37, 38, 61. This 520 linear asymptotic approximation process thus leads to an ${ }_{521}$ artificial energy flux from low frequencies (large scales) 522 to higher frequencies (small scales). It means that the ${ }_{223}$ Fourier-based spectrum may be contaminated by this ar524 tificial energy flux. As another direct consequence, the ificial redistribution of the energy will lead to an un${ }_{526}$ real correlation if we consider cross-correlation between 527 two scales 92 .

The original time series is divided into 122 non529 overlapping segments with $2^{12}$ data points each. The 530 finally spectra and statistical errors (the standard devi531 ation) are then estimated from these 122 realizations. ${ }_{332}$ Figure 9 shows the energy spectra (or the second or533 der statistical moments) provided by HSA (solid line), ${ }_{334}$ Fourier transform (dashed line), WL $(\square)$, the first-order 35 DFA $(\bigcirc)$ and SF $(\diamond)$, respectively. The inset shows the corresponding compensated spectra by multiplying 

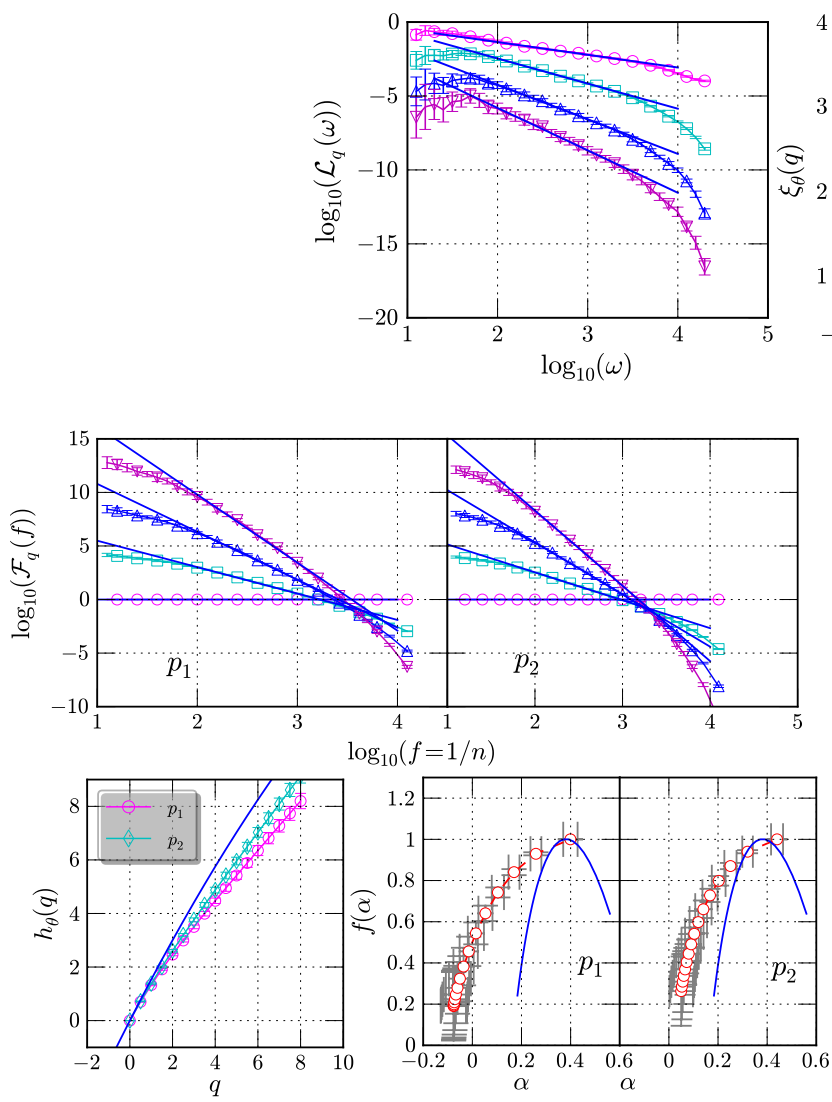

(b)
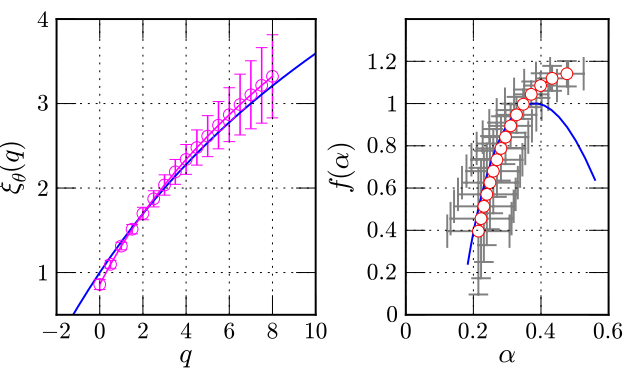

(a)
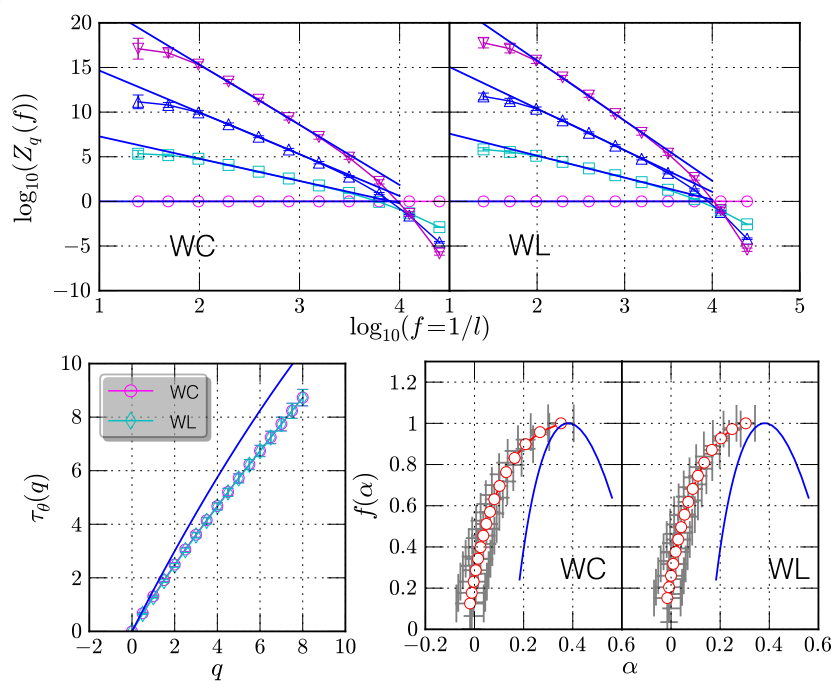

(c)

FIG. 10. (Color online) Results for passive scalar (temperature) with strong ramp-cliff (a) Hilbert spectral analysis, (b) multifractal detrended fluctuation analysis, and (c) wavelet coefficients and wavelet leaders. The symbols are the same as in Fig. 4

${ }_{544}$ for Hilbert, $\beta_{\theta} \simeq 1.56$ for Fourier, $\tau_{\theta}(2) \simeq 2.46$ for ${ }_{568}$ failure of SF analysis, we do not present it here (see ${ }_{545} \mathrm{WL}$ and $h_{\theta}(2) \simeq 2.47$ for DFA, respectively obtained 569 Ref. 33]). Graphically, these three methodologies pre${ }_{546}$ by using a least square fitting algorithm. We note that 570 dict power law spectra with small statistical error. The ${ }_{547}$ only the Hilbert based scaling exponent $\xi_{\theta}(2)$ is close ${ }_{571}$ corresponding scaling exponents are estimated on the ${ }_{548}$ to the corresponding nonintermittent scaling exponent ${ }_{572}$ range $80<f<2000 \mathrm{~Hz}$ or $100<f<1000 \mathrm{~Hz}$. It ${ }_{549} \xi_{\theta}(2)=5 / 3$ [36. It is also comparable with the scaling ${ }_{573}$ is found that the corresponding scaling exponents $\xi_{\theta}(q)$ ${ }_{550}$ exponent of longitudinal velocity in fully developed tur- 574 and singularity spectrum $f(\alpha)$ are close to the lognor${ }_{551}$ bulence 2, 96, 97. Due to the presence of strong ramp- 575 mal fitting model, indicating a less intermittent passive ${ }_{552}$ cliff structures, the SF fails to detect the correct scaling 576 scalar turbulence field than what was believed before [36]. ${ }_{553}$ behavior. The influence of large energetic structures on 577 MFDFA and wavelets provide comparable statistical er${ }_{554} \mathrm{SF}$ has been studied in detail by Huang et al. [36] and 578 rors and singularity spectra. Their scaling exponents and ${ }_{555}$ Huang [33. It is interesting to note that DFA and WL 579 singularity spectra significantly deviate from lognormal 556 provide almost the same scaling exponent, which indi- 580 model, which is usually considered as evidence that the ${ }_{557}$ cates that the ramp-cliff structure may have the same ${ }_{581}$ passive scalar turbulence field is much more intermittent ${ }_{558}$ influence on them. We believe that there exists an arti- 582 than the velocity field 88 91. We note that the first${ }_{559}$ ficial energy flux as we discussed above in both Fourier 583 and second-order MFDFA provide different scaling expo560 and DFA and WL spectra. Thus they may underestimate 584 nents and singularity spectra, which may be associated 561 the scaling exponents [36].

562 Figure 10 shows the analysis results of (a) Hilbert spec585 with the different abilities of different order polynomials 586 [15, 71].

${ }_{563}$ tral analysis, (b) MFDFA, and (c) wavelet transform, ${ }_{587}$ As we already mentioned previously, the wavelet and ${ }_{564}$ respectively. The symbols are the same as in Fig. 4. 588 DFA spectra are strongly influenced by nonlinear large ${ }_{565}$ For comparison, the lognormal model of longitudinal ve- 589 scale structures (e.g. ramp-cliff structures in passive 566 locity 98 is shown as a solid line in the subfigures of 590 scalar turbulence). Their scaling exponents are thus con${ }_{567}$ scaling exponents and singularity spectra. Due to the 591 taminated by high-order harmonics. In other words, the 
592 statistical property of small scales is contaminated by ${ }_{647}$ all the methods require high-order harmonics to represent ${ }_{593}$ nonlinear large-scale structures. We believe here that ${ }_{648}$ the ramp-cliff structures. Therefore, the singularity spec594 the scaling exponents and singularity spectrum provided ${ }_{649}$ tra provided by DFA and WL are contaminated by this 595 by them are not correct. Since the HSA has a very lo- 650 large nonlinear structure. In fact, it already has been 596 cal ability in both physical and spectral spaces, together ${ }_{651}$ reported by several authors that for passive scalar tur${ }_{597}$ with the ability of intrawave-frequency-modulation for ${ }_{652}$ bulence the second-order SF and Fourier power spectrum 598 nonlinear processes, the effect of ramp-cliff structures is 653 are not consistent with each other [90, 99, 100]. Warhaft 599 constrained in the amplitude-frequency space. Therefore, ${ }_{654}$ 90] stated that "the reason for this is unclear, but appar600 the HSA method may provide a more correct scaling ex- 655 ently stems from the Fourier transform itself." There is ${ }_{601}$ ponent and singularity spectrum. We note that for DFA 656 no mathematical transform involved in SF analysis. Now, ${ }_{602}$ and the wavelet method, the large deviation from a log- 657 it seems quite clear that not only Fourier-based methods 603 normal spectrum may be interpreted as a shift problem 658 are strongly influenced by the ramp-cliff structure, but 604 for moment-based methods when the translational invari- 659 also SF analysis [36] and DFA.

605 ance is broken [29, 30. It seems that the Hilbert-based ${ }_{660}$ Our experience is that the HSA is a direct mea606 method can automatically correct this problem. We also ${ }_{661}$ surement of scale-invariant property in the amplitude607 underline here that the Reynolds number of the present ${ }_{662}$ frequency space. It thus requires a much larger sample ${ }_{608}$ passive scalar data set is about $R e_{\lambda} \simeq 250$. Thus the ${ }_{663}$ size to get a convergence result than SFs, DFA and WL. 609 strong ramp-cliff structure may be recognized as an ef- ${ }_{66}$ Thus for a sample of small size without large-scale struc610 fect of the finite Reynolds number. We will address this ${ }_{665}$ tures, SF analysis, DFA or WL are useful to extract scal611 issue elsewhere.

612

\section{CONCLUSION}

613 In summary, we introduced in this paper a new 614 method, namely arbitrary-order Hilbert spectral anal$615 \mathrm{ysis,}$ to characterize scale-invariant properties directly ${ }_{616}$ in the amplitude-frequency space 31 33. It is an ex617 tended version of Hilbert-Huang transform [38, 39, 43]. ${ }_{618}$ The main advantage of the Hilbert-based methodology 619 is its fully adaptive 41 and very local ability both in ${ }_{620}$ spectral and physical domains [38, 39]. Thus, it is not ${ }_{621}$ necessary to require high-order harmonics to represent 622 nonlinear and nonstationary processes, which is usually ${ }_{623}$ required by conventional Fourier-based methods, such as ${ }_{624}$ Fourier transform, wavelet transform, etc. We illustrated 625 the nonlinear effect by using the Duffing equation. It is ${ }_{626}$ found that not only Fourier-based methods, but also SF ${ }_{627}$ analysis and DFA are influenced by nonlinear processes. ${ }_{628}$ It is also found that the HSA can constrain the high-order ${ }_{629}$ harmonics by using the intrawave-frequency-modulation 630 mechanism for the nonlinear distortion [38, 39, 43.

${ }_{631}$ We then performed a comparison study of the Hilbert${ }_{632}$ based methodology with SF analysis, MFDFA, and WL, 633 by analyzing $\mathrm{fBm}$ simulations with Hurst number $H=$ ${ }_{634} 1 / 3$ and a synthesized multifractal lognormal random ${ }^{690}$ ${ }_{635}$ walk with intermittent parameter $\mu=0.15$, respectively. ${ }_{636}$ For the former simulation, we considered the scaling ex${ }_{637}$ ponents and singularity spectrum on the range $0<q<8$. ${ }_{638}$ It was found that all methods provide comparable scaling ${ }_{693}$ No. 11072139) and the Shanghai Program for Innovative ${ }_{639}$ exponents and singularity spectra. For the latter synthe- ${ }_{694}$ Research Team in Universities. Y. H. was financed in 640 sized multifractal random walk data, HSA and MFDFA ${ }_{695}$ part by a grant from the French Ministry of Foreign Af${ }_{641}$ provide a better estimation of singularity spectra than ${ }_{696}$ fairs and in part by Université Lille. Y.H. also acknowl${ }_{642} \mathrm{SF}$ and WL. However, none of these methods recover the ${ }_{69}$ edges a financial support from EHL of Université Libre de ${ }_{643}$ whole spectrum. We finally applied all methods to the ${ }_{698}$ Bruxelles during the preparation of this manuscript. We ${ }_{644}$ passive scalar (temperature) data set with strong ramp- ${ }_{699}$ thank Professor P. Abry from Laboratoire de Physique, ${ }_{645}$ cliff structure, which is an important signature of passive ${ }_{700}$ CNRS and ENS Lyon (France) for providing his wavelet ${ }_{646}$ scalar turbulence [89, 90. We found that except for HSA, 701 leader codes. The EMD Matlab codes used in this paper 
702 are written by Dr. Gabriel Rilling and Professor Patrick 703 Flandrin from Laboratoire de Physique, CNRS and ENS 704 Lyon (France): 101.

[1] F. Anselmet, Y. Gagne, E. J. Hopfinger, and R. A. 762 Antonia, J. Fluid Mech. 140, 63 (1984).

[2] U. Frisch, Turbulence: the legacy of AN Kolmogorov 764 (Cambridge University Press, 1995).

[3] D. Lohse and K.-Q. Xia, Ann. Rev. Fluid Mech. 42, 335766 (2010).

[4] D. Schertzer and S. Lovejoy, J. Geophys. Res 92, 9693768 (1987).

[5] F. G. Schmitt, S. Vannitsem, and A. Barbosa, J. Geo- 770 phys. Res. 103, 23181 (1998).

[6] M. De Lima and J. Grasman, J. Hydrol. 220, 1 (1999). 772

[7] V. Venugopal, S. G. Roux, E. Foufoula-Georgiou, and 773 A. Arnéodo, Phys. Lett. A 348, 335 (2006).

[8] S. Ghashghaie and Y. Dodge, Nature 381, 27 (1996). 775

[9] F. G. Schmitt, D. Schertzer, and S. Lovejoy, Appl. 776 Stoch. Models and Data Anal. 15, 29 (1999).

10] T. Lux, Quantitative Finance 1, 560 (2001).

11] L. Calvet and A. Fisher, Review of Economics and 77 Statistics 84, 381 (2002).

[12] P. Ivanov, A. Bunde, L. Amaral, S. Havlin, J. Fritsch- 781 Yelle, R. Baevsky, H. Stanley, and A. Goldberger, Eu- 782 rophys. Lett. 48, 594 (1999).

[13] A. S. Monin and A. M. Yaglom, Statistical fluid me- 78 chanics vd II (MIT Press Cambridge, Mass, 1971). $\quad 785$

[14] C.K. Peng, S.V. Buldyrev, S. Havlin, M. Simons, 786 H.E. Stanley, and A.L. Goldberger, Phys. Rev. E 49, 787 1685 (1994).

[15] K. Hu, P.C. Ivanov, Z. Chen, P. Carpena, and H.E. Eu- 789 gene Stanley, Phys. Rev. E 64, 11114 (2001).

16] A Bashan, R. Bartsch, J. Kantelhardt, and S. Havlin, Physica A 387, 5080 (2008). S. Havlin A. Bunde, and H. Stanley, Physica A 316, 87 (2002).

[18] J.F. Muzy, E. Bacry, and A. Arneodo, Phys. Rev. Lett. 796 67, 3515 (1991).

[19] J.F. Muzy, E. Bacry, and A. Arneodo, Phys. Rev. E 798 47, 875 (1993).

[20] J. Arrault, A. Arneodo, A. Davis, and A. Marshak, 800 Phys. Rev. Lett. 79, 75 (1997).

[22] M. Farge, N. Kevlahan, V. Perrier, and E. Goirand, IEEE J PROC 84, 639 (1996).

[23] M. Farge, Annu. Rev. Fluid Mech. 24, 395 (1992). 806

[24] J. Ghez and S. Vaienti, J. Statist. Phys. 57, 415 (1989). 807

[25] S. Jaffard, B. Lashermes, and P. Abry, Wavelet Analysis 808 and Applications (2005).

[26] B. Lashermes, S. Roux, P. Abry, and S. Jaffard, EPJB 61, 201 (2008).

27] E. Serrano and A. Figliola, Physica A 388, 2793 (2009). 812

[28] B. Lashermes, S. Jaffard, and P. Abry, ICASSP 2005, 813 Philadelphia, USA, (2005).

[29] O. Pont, A. Turiel, and C. Pérez-Vicente, Physical Re- 815 view E 74, 061110 (2006).

[30] A. Turiel, C.J. Pérez-Vicente, and J. Grazzini, J. Com- 817 put. Phys. 216, 362 (2006).
[31] Y. Huang, F. G. Schmitt, Z. Lu, and Y. Liu, Europhys. Lett. 84, 40010 (2008).

[32] Y. Huang, F. G. Schmitt, Z. Lu, and Y. Liu, Traitement du Signal 25, 481 (2008).

[33] Y. Huang, Arbitrary Order Hilbert Spectral Analysis: Definition and Application to fully developed turbulence and environmental time series, Ph.D. thesis, Université des Sciences et Technologies de Lille - Lille 1, France \& Shanghai University, China (2009);http://tel.archivesouvertes.fr/tel-00439605/fr..

[34] F. G. Schmitt, Y. Huang, Z. Lu, L. Y., and N. Fernandez, J. Mar. Sys. 77, 473 (2009).

[35] Y. Huang, F. G. Schmitt, Z. Lu, and Y. Liu, J. Hydrol. 373, 103 (2009).

[36] Y. Huang, F. Schmitt, Z. Lu, P. Fougairolles, Y. Gagne, and Y. Liu, Phys. Rev. E 82, 26319 (2010).

[37] L. Cohen, Time-frequency analysis (Prentice Hall PTR Englewood Cliffs, NJ, 1995).

[38] N. E. Huang, Z. Shen, S. R. Long, M. C. Wu, H. H. Shih, Q. Zheng, N. Yen, C. C. Tung, and H. H. Liu, Proc. R. Soc. London, Ser. A 454, 903 (1998).

[39] N. E. Huang, Z. Shen, and S. R. Long, Annu. Rev. Fluid Mech. 31, 417 (1999).

[40] G. Rilling, P. Flandrin, and P. Gonçalvès, IEEEEURASIP Workshop on Nonlinear Signal and Image Processing (2003).

[41] P. Flandrin and P. Gonçalvès, Int. J. Wavelets, Multires. Info. Proc. 2, 477 (2004).

[42] N. E. Huang, M. L. Wu, S. R. Long, S. S. P. Shen, W. Qu, P. Gloersen, and K. L. Fan, Proc. R. Soc. London, Ser. A 459, 2317 (2003).

[43] N. E. Huang, Hilbert-huang transform and its applications, (World Scientific, Singapore, 2005) Chap. 1, pp. $1-26$.

[44] P. A. Hwang, N. E. Huang, and D. W. Wang, Appl. Ocean Res. 25, 187 (2003).

[45] A. D. Veltcheva and C. G. Soares, Appl. Ocean Res. 26, 1 (2004).

[46] J. C. Echeverria, J. A. Crowe, M. S. Woolfson, and B. R. Hayes-Gill, Med. Biol. Eng. Comput. 39, 471 (2001).

[47] R. Balocchi, D. Menicucci, E. Santarcangelo, L. Sebastiani, A. Gemignani, B. Ghelarducci, and M. Varanini, Chaos Soliton Fract. 20, 171 (2004).

[48] V. I. Ponomarenko, M. D. Prokhorov, A. B. Bespyatov, M. B. Bodrov, and V. I. Gridnev, Chaos Soliton Fract. 23, 1429 (2005).

[49] N. E. Huang, M. L. Wu, W. Qu, S. R. Long, and S. S. P. Shen, Appl. Stoch. Model Bus. 19, 245 (2003).

[50] K. T. Coughlin and K. K. Tung, Adv. Space Res. 34, 323 (2004).

[51] I.M. Jánosi and R. Müller, Phys. Rev. E 71, 56126 (2005).

[52] M. K. I. Molla, M. S. Rahman, A. Sumi, and P. Banik, Discrete Dyn. Nat. Soc. 2006, Article ID 45348, 17 pages (2006), doi:10.1155/DDNS/2006/45348. 
[53] J. Solé, A. Turiel, and J. Llebot, Nat. Hazard Earth 867 Sys. Sci. 7, 299 (2007).

54] Z. Wu, N. E. Huang, S. R. Long, and C. Peng, PNAS ${ }_{86}$ 104, 14889 (2007).

[55] C. H. Loh, T. C. Wu, and N. E. Huang, BSSA 91, 1339 (2001).

[56] J. Chen, Y. L. Xu, and R. C. Zhang, J. Wind Eng. Ind. 873 Aerodyn. 92, 805 (2004).

[57] S. J. Loutridis, Appl. Acoust. 66, 1399 (2005).

58] F. G. Schmitt, Y. Huang, Z. Lu, S. B. Zongo, J. C. 876 Molinero, and Y. Liu, in Nonlinear Dynamics in Geo- 877 sciences. edited by A. Tsonis and J. Elsner (Springer, 878 2007) pp. 261-280.

59] P. Flandrin, G. Rilling, and P. Gonçalvès, IEEE Sig. Proc. Lett. 11, 112 (2004).

60] S. R. Long, N. E. Huang, C. C. Tung, M. L Wu, R. Q. Lin, E. Mollo-Christensen, and Y. Yuan, IEEE Geo- 883 science and Remote Sensing Soc. Lett. 3, 6 (1995). 884

[61] P. Flandrin, Time-frequency/time-scale analysis (Aca- 885 demic Press, 1998).

[62] In fact, the Eq. 16 is convergence when $q \geq-1$. How- 88 ever, in practice, we only consider the case $q \geq 0$.

63] G. Rilling and P. Flandrin, IEEE International Confer- 88 ence on Acoustics, Speech and Signal Processing, 2006. 890 ICASSP 2006 Proceedings. 2006 3, 444 (2006).

64] G. Rilling and P. Flandrin, IEEE Trans. Signal Process (2008).

[65] G. Rilling and P. Flandrin, Adv. Adapt. Data Anal. 1, 894 43 (2009).

[66] D. Schertzer, S. Lovejoy, F. G. Schmitt, Y. Chigirin- 896 skaya, and D. Marsan, Fractals 5, 427 (1997).

[67] J. Schmittbuhl, F. G. Schmitt, and C. Scholz, J. geo- 89 phys. Res 100, 5953 (1995).

[68] F. G. Schmitt, S. Lovejoy, and D. Schertzer, Geophys. 900 Res. Lett. 22, 1689 (1995).

69] P. Oświęcimka, J. Kwapień, and S. Drożdż, Phy. Rev. 902 E 74, 16103 (2006).

70] C. Heneghan and G. McDarby, Phys. Rev. E 62, 6103 (2000). Rev. E 65, 041107 (2002).

[72] E. Koscielny-Bunde, J. Kantelhardt, P. Braun, 908 A. Bunde, and S. Havlin, J. Hydrol. 322, 120 (2006). 909

[73] M. Sadegh Movahed, G. Jafari, F. Ghasemi, S. Rahvar, 910 and M. Rahimi Tabar, J. Stat. Mech. , 02003 (2006).

[74] J. Bardet and I. Kammoun, Information Theory, IEEE Transactions on 54, 2041 (2008).

75] Q. Zhang, C. Xu, Y. Chen, and Z. Yu, Hydrol. Process. 22, 4997 (2008).
[76] S. Mallat and W. Hwang, IEEE T. Inform. Theory. 38, 617 (1992).

[77] S. Mallat, A wavelet tour of signal processing (Academic Pr, 1999).

[78] H. Wendt, P. Abry, and S. Jaffard, IEEE Signal Processing Mag. 24, 38 (2007).

[79] I. Daubechies, Ten lectures on wavelets (Philadelphia: SIAM, 1992).

[80] J. Beran, Statistics for long-memory processes (CRC Press, 1994).

[81] L. Rogers, Math. Finance 7, 95 (1997).

[82] P. Doukhan, M. Taqqu, and G. Oppenheim, Theory and Applications of Long-Range Dependence (Birkhauser, 2003).

[83] C. W. Gardiner, Handbook of Stochastic Methods (Springer, Berlin, third edition, 2004).

[84] A. Wood and G. Chan, J. Comput. Graph. Stat. 3, 409 (1994).

[85] F. G. Schmitt, Eur. Phys. J. B 34, 85 (2003).

[86] E. Bacry, J. Delour, and J.F. Muzy, Phys. Rev. E 64, 026103 (2001).

[87] J.F. Muzy and E. Bacry, Phys. Rev. E 66, 056121 (2002).

[88] K. Sreenivasan, Proc. R. Soc. Lond. A 434, 165 (1991).

[89] B. Shraiman and E. Siggia, Nature 405, 639 (2000).

[90] Z. Warhaft, Annu. Rev. Fluid Mech. 32, 203 (2000).

[91] A. Celani, A. Lanotte, A. Mazzino, and M. Vergassola, Phys. Rev. Lett. 84, 2385 (2000).

[92] Y. Huang, F. G. Schmitt, and Y. Gagne, in preparation for Phys. Rev. Lett..

[93] A. N. Kolmogorov, Dokl. Akad. Nauk SSSR 30, 301 (1941).

[94] A. Obukhov, Izv. Acad. Nauk SSSR Ser. Geog. Geofiz 13, 58 (1949).

[95] S. Corrsin, J. Appl. Phys. 22, 469 (1951).

[96] Z. S. She and E. Lévêque, Phys. Rev. Lett. 72, 336 (1994).

[97] W. van de Water and J. A. Herwijer, J. Fluid Mech. 387, 3 (1999).

[98] F. G. Schmitt, Physica A 368, 377 (2006).

[99] R.A. Antonia, E.J. Hopfinger, Y. Gagne, and F. Anselmet, Phys. Rev. A 30, 2704 (1984).

[100] G. Ruiz-Chavarria, C. Baudet, and S. Ciliberto, Physica D 99, 369 (1996).

[101] Http://perso.ens-lyon.fr/patrick.flandrin/emd.html. 